\title{
Las razones del cambio de desempeño laboral en los docentes de Educación Física en el contexto gallego \\ The reasons for changing jobs among Physical Education teachers in the Galician context
}

*Raúl Eirín Nemiña, **Alberto Sanmiguel-Rodríguez, *Jesús Rodríguez Rodríguez

*Universidad de Santiago de Compostela (España), ** Universidad Camilo José Cela (España) **Universidad Antonio de Nebrija (España)

Resumen. En este estudio analizamos las razones que justifican los cambios de desempeño laboral en los docentes de Educación Física (EF). De un modo concreto, nos preguntamos por las razones del desgaste profesional que sufren los docentes y que justifican los cambios y abandono de los docentes. Para ello desarrollamos un estudio descriptivo en el que participaron un total de 120 docentes de EF que realizan su función en las etapas de Educación Primaria y Secundaria en la Comunidad Autónoma de Galicia. Para la obtención de información cumplimentaron un cuestionario, elaborado a partir del Teacher Follow-up Survey. Destacamos que, en general, el profesorado indica que laescasa valoración hacialaEF por par te de la administración influye mucho en dicho cambio de trabajo. Igualmente, otro de los factores determinantes del cambio de desempeño laboral es la escasa consideración hacia la EF por parte de las familias. Los docentes cuando deciden buscar un nuevo horizonte profesional suelen optar por un cambio de especialidad y la dedicación a tareas de gestión docente.

Palabras clave: desempeño laboral, especialidad docente, educación física.

\begin{abstract}
In this study we analyze the reasons that justify changes in job performance in Physical Education (PE) teachers. In a concrete way, we ask ourselves about the reasons for the professional burnout suffered by teachers and that justify the changes and abandonment of teachers. For this, we developed a descriptive study in which a total of $120 \mathrm{PE}$ teachers participated, who perform their function in the stages of Primary and Secondary Education in theAutonomous Community of Galicia. To obtain information, they completed a questionnaire, prepared from theTeacher Follow-up Survey. We highlight that, in general, the teaching staff indicates that the low assessment of PE by the administration greatly influences this job change. Likewise, another of the determining factors of the change in job performance is the scant consideration for PE by families.W hen teachers decide to seek a new professional horizon, they usually opt for a change of specialty and dedication to teaching management tasks.
\end{abstract}

Keywords: job performance, teaching specialty, physical education.

\section{Introducción}

El desempeño de la profesión docente implica un cierto desgaste y erosión del entusiasmo que acompaña a los docentes en sus inicios profesionales. Para comprender esta situación conviene poner de relieve, atendiendo a lo que recoge la literatura como uno de los aspectos clave, el significado o la influencia de la satisfacción profesional. En este sentido, la satisfacción con el trabajo que se realiza es un predictor importante del desempeño profesional. La definición clásica de Locke (1976) presenta la satisfacción profesional como el estado emocional positivo y placentero que experimenta una persona, como resultado de la percepción subjetiva de sus propias experiencias laborales. Spector (1996) habla de la

Fecha recepción: 03-07-20. Fecha de aceptación: 07-12-20 Alberto Sanmiguel-Rodríguez asrgz2014@gmail.com satisfacción profesional como el grado de disfrute de una persona con su trabajo, y en la misma línea, Weiss (2002) la define como una actitud basada en los juicios positivos o negativos de una persona hacia su actividad laboral. Así, una visión positiva del trabajo puede asociarse con la satisfacción profesional, mientras que si esta visión es negativa se puede hablar de insatisfacción laboral. Más allá de esta imagen dicotómica, es importante verlas como polos de un continuo (Pinder, 1998) que van evolucionando con las circunstancias y estadios de desarrollo del profesional.

La literatura recoge, al menos, dos tipologías de satisfacción profesional (Judge, Thoresen, Bono y Patton, 2001; Skaalvik y Skaalvik, 2011): por un lado la satiffacción general con el trabajo y, por el otro, aquellos aspectos o factores específicos que contribuyen a hacerlo satisfactorio. Dentro de estos factores se señalan, entre otros, los siguientes: condiciones laborales precarias (Buckley, Schneider y Shang, 
2004; Hancock y Scherff, 2010); falta de recursos (Buckley et al., 2004); problemas en la gestión del centro (Ingersoll y Merrill, 2010); dificultades con el comportamiento del alumnado y la atención a la diversidad (M acBeath, 2012; M oore, 2012); intensificación de la carga burocrática asociada (Barmby, 2006; Perrachione, Rosser y Petersen, 2008) falta de autonomía y desprofesionalización (M ihans, 2008; Zembylas y Papanastasiou, 2006) y falta de cultura colaborativa en el centro (H argreaves, 2019; K arsenti y Collin, 2013).

En el trabajo de Á valos y Valenzuela (2016) sobre los argumentos que esgrimen los docentes para abandonar la profesión, aparecen tres razones principales: la falta de oportunidades de desarrollo profesional, las precarias condiciones laborales y los problemas de gestión de la escuela. Concretamente, los aspectos más señalados, en orden decreciente de importancia son: 1. Insatisfacción con las oportunidades de desarrollo profesional. 2. Remuneración insuficiente. 3. Falta de beneficios complementarios adicionales adecuados. 4. Insatisfacción con el liderazgo en la gestión de la escuela. 5. Insatisfacción con las condiciones materiales de la escuela. 6 . Falta de influencia sobre las políticas y prácticas de la escuela. 7. Deseo de mejorar el estándar de vida. 8. Insuficiente autonomía para tomar decisiones pedagógicas. 9. Deseo de seguir formándose para tener más oportunidades en el campo educativo.10. Número de estudiantes demasiado alto.

Por otra parte, encontramos en la literatura en Educación Física (EF) una serie de razones que generan cierto malestar y desgaste, lo que provoca, en algunos casos, abandono de la profesión. El estatus marginal que se le da a la EF por parte de los propios colegas docentes (Hardman, 2011) y su consideración como materia sin contenido académico, como actividad recreativa (M acdonald, 1995; Moreira, Sparkes y Fox, 1995), no ayudan a mejorar su consideración académica y social. Esta falta de valoración y reconocimiento mina la autoestima de los docentes de EF e influye negativamente en su labor docente (Gaudreault yWoods, 2013). Por otra parte, cuando son reconocidos como buenos docentes se les asignan otras tareas de «mayor importancia», Io que indirectamente sigue marginando el status de la materia (Smith y Leng, 2003). Richards y Templin (2012) señalan como característico de la profesión la confusión entre las identidades como docente y como entrenador, entre los valores educativos y los deporti- vos, no siempre coincidentes. Añaden a esta situación la sobrecarga de trabajo asociada, las responsabilidades sobre la seguridad de sus estudiantes en entornos y actividades con riesgos potenciales de accidentes, la responsabilidad sobre la salud y condición física de su alumnado. También se incluyen aspectos como la propia presión social sobre su propio cuerpo y el del alumnado (Martínez y González, 2016). Igualmente se señala el aislamiento (Mohr y Townsend, 2001) dentro de las estructuras escolares (físico y mental) al trabajar en espacios apartados, con metodologías y contenidos muy diferentes a las demás materias escolares, con pocos o ningún compañero dentro de la escuela con los que compartir y aprender como un elemento que no ayuda a mejorar la satisfacción de los docentes. De igual manera, tal y como apuntan (Sargent y Hannum, 2005), es importante identificar las razones que están detrás de la desafección de los docentes con la profesión, para prevenir situaciones de absentismo y abandono de la profesión. Resulta de interés, igualmente, conocer el nivel de satisfacción de los docentes de EF, y también la insatisfacción, por encontrarse en el origen de las justificaciones de abandono de la docencia.

A la luz de las reflexiones apuntadas, se pretende conocer las razones del desgaste que sufren los docentes y que justifican el cambio de desempeño laboral en su trayectoria profesional. De un modo concreto, en este artículo se busca dar explicación a la siguiente cuestión central: ¿Cuáles son las razones del desgaste que sufren los docentes y que justifican las intenciones de cambio/ abandono de la profesión?

\section{Metodología}

\section{Participantes}

En el estudio descriptivo participaron un total de 120 docentes en EF que realizan su función en las etapas de Educación Primaria y Secundaria en la Comunidad Autónoma (CCAA) de Galicia, de los cuales un $70,8 \%$ son hombres (85) y el restante $29,2 \%$ mujeres (35). Las edades de dichos docentes comprendían entre los 26 y los 66 años. Los años de experiencia docente en la materia de EF fueron desde un curso académico hasta 36 años. Según la titularidad del centro, el 84,2\% trabajan en centros de enseñanza públicos, un $15 \%$ en centros concertados y un $0,8 \%$ en centros privados. Para la obtención de información cumplimentaron un cuestionario que es una versión del Teacher Follow-up Survey (Keigher, 
2010), traducido al gallego y presentado a dos expertos para su corrección y validación.

\section{Instrumento}

El cuestionario consta de 115 preguntas divididas en 5 secciones. Las secciones del cuestionario se dividieron de la siguiente manera (Tabla 1). Todos los factores del estudio fueron tratados como variables continuas, y el formato de respuesta empleado en todos los instrumentos fue una escala tipo Likert en la que cada ítem tenía un rango de respuesta de 1 a 5. El 1 se correspondía con muy poco satisfecho y el 5 con muy satisfecho de acuerdo con la formulación de la pregunta. Para algunas preguntas el rango de las respuestas fue de 1 a 3 , siendo 1 poco de satisfecho/ no adecuado y 3 de satisfecho/ adecuado. Asimismo, en algunos ítems, dada la formulación de la pregunta, se dejó como respuesta abierta.

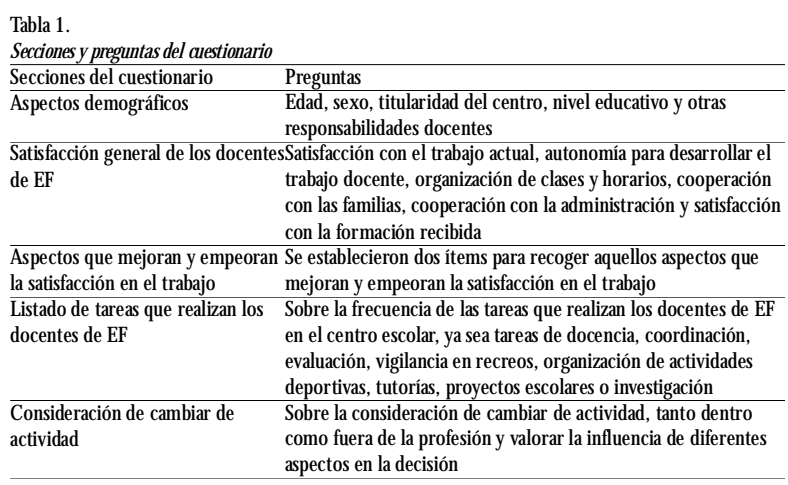

Para comprobar la adecuación del instrumento también se ha analizado su fiabilidad a partir del estudio del Alfa de Cronbach. En esencia, este cálculo indica si el cuestionario es consistente internamente y si mide lo que efectivamente pretende medir. En este caso, el valor obtenido para el conjunto del cuestionario es de 0,818 , lo que supone un elevado grado de fiabilidad (el rango del estadístico se sitúa entre 0 y 1). Con el objetivo de analizar esta cuestión de forma pormenorizada, se analizaron por separado las dos grandes dimensiones del cuestionario: satisfacción con el trabajo y deseos de cambio de empleo, obteniendo, en ambos casos, valores aún más elevados (0,929 y 0,954, respectivamente). La consistencia interna del cuestionario es, pues, muy elevada, con unos valores altamente homogéneos para cada una de las variables empleadas.

\section{Procedimiento}

En primer lugar se procedió a enviar una carta a los centros educativos públicos y privados-concerta- dos de la CCAA de Galicia, tanto de primaria como de secundaria. En ella se explican los motivos de la investigación y la invitación a participar en la misma. Se incluye un enlace a la encuesta y el encargo de que se traslade a los docentes de EF del centro. Dicha encuesta se subió a Google Drive y permaneció activa durante dos meses. Se realizó un seguimiento de las respuestas recibidas y dos semanas antes del cierre de la misma se envió un recordatorio de participación a aquellos centros que no lo habían hecho. Así pues, la primera fase consistió en un estudio transversal de carácter cuantitativo basado en la administración de un cuestionario a una muestra representativa de centros de educación primaria y secundaria, tanto públicos como concertados o privados para docentes de EF en la CCAA de Galicia.

\section{Análisis estadístico}

Para el análisis cuantitativo se empleó el paquete estadístico SPSS, debido a su alta capacidad y eficiencia en el tratamiento y análisis de los datos. Inicialmente se realizó un análisis exploratorio general de los resultados a partir de un análisis descriptivo, especialmente frecuencias y estadísticos. Este primer paso sirvió para conocer de forma preliminar los datos obtenidos y, en consecuencia, poder identificar e indagar más acerca de los aspectos de mayor interés para la investigación.

A modo de contextualización y justificación se observó que el $5 \%$ de los docentes estudiados afirmaron estar insatisfechos con su trabajo actual. Q uienes así contestaron son mayoritariamente hombres, con una media de edad de 43,2 años y una experiencia profesional promedio de 18 años. Por su parte, las personas que se han planteado en al gún momento cambiar de empleo 0 de puesto de trabajo suponen más de un tercio de la población estudiada (37\%) y cuentan con unas características semejantes a las de las personas insatisfechas con su trabajo $(45,8$ años de media de edad y 18 años de experiencia profesional).

Para identificar a las personas con intenciones de cambio en el cuestionario se plantearon dos variables distintas: «¿Desde hace un año consideraste cambiar tu trabajo actual en la EF por otro puesto?» p71- (planteada como pregunta abierta) y «iDesde hace un año, consideraste cambiar tu trabajo actual en la EF por otro trabajo fuera del ámbito escolar?» - p72- (medida en una escala ordinal de 1 (N unca) a 4 (Con frecuencia). Para trabajar con estas variables 
se han seguido los siguientes pasos:

-En primer lugar, se recodificó la p71 para crear una nueva variable dicotómica con la que trabajar de forma más eficiente.

-A continuación, se creó una nueva variable dicotómica a partir de la p72, de tal manera que se dividió a la población en dos categorías: aquellas personas que nunca se plantearon un cambio y aquellas que alguna vez (por pocas que fuesen) sí se lo llegaron a plantear. La creación de esta variable no implica que se haya desechado la variable desagregada original, sino que se han empleado ambas de forma complementaria.

-Finalmente, se creó una variable que combina las dos anteriores: «¿D esde hace un año consideraste cambiar de puesto de trabajo en la EF por otro 0 consideraste cambiar a otro empleo fuera del ámbito escolar?»

Estas variables han sido las que han articulado el análisis de la intención de cambio de trabajo y son las que se han empleado, en general, para establecer las relaciones con el resto de variables del cuestionario.

Debido a la naturaleza categórica de las variables empleadas, las relaciones entre ellas se han representado a partir de tablas de contingencia y se ha empleado el estadístico Chi-cuadrado para medir su grado de dependencia o independencia. Así, por ejemplo, el grado de satisfacción está relacionado con la intención de cambio combinada, como era de esperar $\left(\div^{2}=15,223\right.$, con un valor de significación de 0,04). En este sentido, se han analizado, no sólo las relaciones entre la variable creada ad hoc, sino también las de cada una por separado (p71 y p72) y se han identificado matices de interés en los resultados de una y otra. Para el análisis de las variables numéricas (edad, horas de docencia 0 años de experiencia) se utilizó AN OVA, ofreciendo, por lo general, resultados estadísticamente poco significativos.

\section{Resultados}

En este apartado presentamos los resultados relacionados con las razones de desgaste que sufren los docentes y que están detrás de las intenciones de cambio/ abandono de la profesión de los docentes de EF. A modo de contextualización inicial, en laTabla 2 se puede ver el conjunto de los factores sobre los que se ha interrogado al profesorado participante, así como las respuestas proporcionadas. Veamos a continuación de un modo más detallado los resultados ob-
Tabla 2.

Factores relacionados con la satiffacción y el desgaste de los docentes de EF

Elementos $y$ factores Adecuado Regular No adecuado

\begin{tabular}{lccc} 
Elementos y factores & $\begin{array}{c}\text { Adecuado } \\
\text { / Bueno }\end{array}$ & $\begin{array}{r}\text { Regular No adecuado } \\
\text { / mala }\end{array}$ \\
\hline Instalaciones deportivas & $39,1 \%$ & $27,5 \%$ & $33,4 \%$ \\
M antenimiento instalaciones & $40,8 \%$ & $25,8 \%$ & $33,4 \%$ \\
Calidad de las instalaciones & $40,8 \%$ & $25,8 \%$ & $33,4 \%$ \\
Material & $50 \%$ & $24,2 \%$ & $25,8 \%$ \\
\hline Consideración general de la EF & $40 \%$ & $24,2 \%$ & $35,8 \%$ \\
Consideración de otros docentes hacia la EF & $37,5 \%$ & $35 \%$ & $27,5 \%$ \\
Colaboración con otros docentes & $60,8 \%$ & $25,8 \%$ & $13,3 \%$ \\
Consideración de la administración sobre la EF & $9,1 \%$ & $31,7 \%$ & $59,2 \%$ \\
Cooperación con la administración & $23,3 \%$ & $37,5 \%$ & $39,2 \%$ \\
Colaboración con las actividades deportivas municipales & $34,1 \%$ & $32,5 \%$ & $33,4 \%$ \\
Colaboración con agrupaciones deportivas & $33,3 \%$ & $38,3 \%$ & $28,4 \%$ \\
Cooperación con los padres y/ o madres & $42,5 \%$ & $35,8 \%$ & $21,7 \%$ \\
Respeto de los padres y/ o madres hacia la EF & $27,5 \%$ & $33,3 \%$ & $39,2 \%$ \\
\hline Organización de horarios & $64,2 \%$ & $20,8 \%$ & $15 \%$ \\
Número de horas de docencia & $56,6 \%$ & $24,2 \%$ & $19,2 \%$ \\
Ratio de alumnos/ as & $54,2 \%$ & $20 \%$ & $25,8 \%$ \\
Comportamiento y actitud del al umnado hacia la EF & $55,9 \%$ & $29,2 \%$ & $14,9 \%$ \\
Colaboración con los al umnos/ as & $78,3 \%$ & $17,5 \%$ & $4,2 \%$ \\
\hline
\end{tabular}

tenidos.

Respecto a las instalaciones y equipamientos, se puede observar que no suponen un factor de desgaste importante en las opiniones de los docentes. La valoración entre buena y regular se sitúa alrededor del $70 \%$, siendo las valoraciones hacia el material negativas en el $25 \%$.

Respecto a la organización docente, ofrecen la mejor valoración, situándose en val ores entre el $70 \%$ y $80 \%$, siendo el factor con peor valoración el relacionado con la ratio de alumnos $(25,8 \%)$. Por el contrario, los factores relacionados con la colaboración y la consideración hacia la EF, presentan unos porcentajes de valoración negativa muy superiores. Así pues, los aspectos que más influyen inicialmente en el deseo de cambio guardan mucha relación con la percepción que se tiene de la Administración por parte de los docentes. Así, los cinco aspectos que han influido en mayor medida son: falta de apoyo de la Administración $(66,7 \%$ dicen que influyen mucho 0 muchísimo $\left.{ }^{1}\right)$, falta de confianza en laAdministración $(61,9 \%)$, incompetencia de la Administración $(57,1 \%)$, falta de respeto y valoración de la Administración hacia la EF $(52,4 \%)$ y rigidez de la Administración $(52,4 \%)$. Parece claro, pues, que una percepción negativa de la Administración gallega tiene un papel determinante a la hora de plantearse cambiar 0 no de puesto de trabajo (Figura 1). En el sentido opuesto, el ambiente de trabajo no ha influido prácticamente nada en la intención de cambio. Específicamente, el 90,5\% del profesorado que desea cambiar dice que la mala relación con los compa-

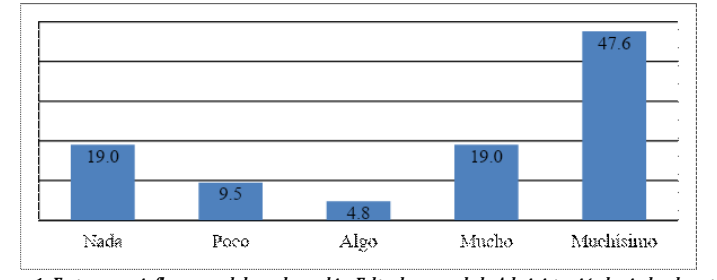

Figura 1. Factores que influyen en el deseo de cambio: Falta de apoyo de la Administración hacia los docentes 
ñeros ha influido poco o nada en su deseo, el 85,7\% dice lo mismo del aislamiento respecto a los compañeros y el $75 \%$ lo afirma respecto a las envidias profesionales. Paralelamente, el $80 \%$ indica que el deseo de mayor autonomía laboral ha influido poco 0 nada en esta cuestión.

En cualquier caso, quienes se han planteado un cambio en este sentido (18,3\% del total de docentes) optarían mayoritariamente por ejercer como maestro/ a generalista $(40,9 \%)$ o por enseñar otra materia (27,3\%). Tan sólo al $13,6 \%$ le gustaría formar parte del equipo directivo del centro.

En cuanto a sus características sociodemográficas y profesionales, no hay apenas diferencias entre las personas que no se han planteado un cambio y quienes sí lo han hecho: no hay diferencia por género (el $18,8 \%$ de los hombres y el $17,7 \%$ de las mujeres quieren cambiar), ni por edad (misma edad promedio, 46), ni por años de experiencia docente $(17,5$ y 16,4, respectivamente). Hay, no obstante, dos aspectos que arrojan diferencias que, sin ser estadísticamente significativas, pueden suponer un indicio de la realidad: los docentes de centros públicos y los que tienen otras responsabilidades además de la docencia en EF parecen más dispuestos a cambiar de puesto de trabajo (Tabla 3).

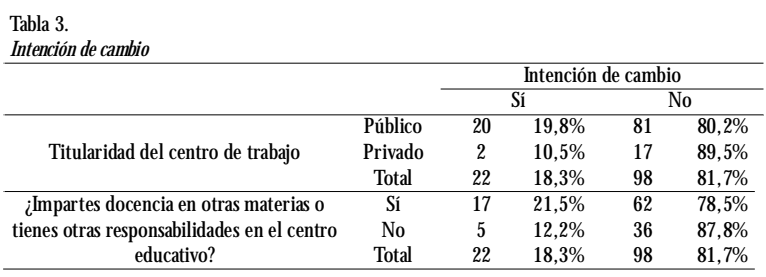

Con respecto a las intenciones de cambio de empleo fuera del ámbito de la educación, el volumen de personas que se lo han planteado supone el $25,8 \%$ sobre el total de docentes que han respondido al cuestionario. Las diferencias entre las personas que se han planteado un cambio de profesión y las que no, son, nuevamente, mínimas: ni el género, la edad, la experiencia docente 0 la titularidad del centro de trabajo discriminan entre uno y otro grupo. No obstante, el hecho de tener más responsabilidades además de la docencia en EF sí que guarda relación con la intención de cambio: las personas que no son sólo

Tabla 4

Intenciones de cambio de empleo fuera del ámbito de la educación

\begin{tabular}{cccccc}
\hline & \multicolumn{4}{c}{ Intención de cambio } \\
\cline { 2 - 6 } & \multicolumn{4}{c}{ Sí } & \multicolumn{1}{c}{ No } \\
\hline \multirow{2}{*}{ ¿Impartes docencia en otras materias o tienes } & Sí & 25 & $31,6 \%$ & 54 & $68,4 \%$ \\
otras responsabilidades en el centro educativo? & No & 6 & $14,6 \%$ & 35 & $85,4 \%$ \\
& Total & 31 & $25,8 \%$ & 89 & $74,2 \%$ \\
\hline \multirow{2}{*}{$\left(x^{2}=4,077\right.$, con un valor de significación de 0,43$)$}
\end{tabular}

docentes de EF, presentan intenciones de cambio en mayor proporción $(31,6 \%$ y $14,6 \%)$ tal y como se muestra en la tabla 4.

Los factores que más influyen en el deseo de cambio de empleo son las mismas que en el caso de cambio de puesto de trabajo. Así, la falta de apoyo de la Administración influye mucho o muchísimo para el $67,9 \%^{2}$, y tanto la falta de respeto y de valoración por parte de laAdministración como la falta de confianza en ella son determinantes para el 60,7\%.También tienen gran peso la incompetencia $(57,1 \%)$ y la rigidez de la Administración (51,9\%). Los aspectos menos influyentes también son los mismos que en el caso anterior, si bien con ligeros matices en el orden 0 en el porcentaje. Gran parte de las personas que pensaron en cambiar la educación por otro empleo lo harían para dedicarse a cuestiones relacionadas con el deporte: entrenador deportivo $(31,8 \%)$ o gestión deportiva (13,6\%). No obstante, el $18,2 \%$ lo cambiaría por cualquier otro empleo sin relación alguna ni con el deporte ni con la educación.

En general, las personas que han pensado en cambiar de puesto de trabajo dentro de la educación y las personas que se han planteado cambiar de empleo por otro fuera de la educación (que no siempre son las mismas) tienen prácticamente las mismas características y en sus intenciones influyen los mismos factores. Sin embargo, al relacionar ambas variables con el grado de satisfacción al respecto de distintos aspectos laborales, se pueden observar diferencias de cierto calado: la intención de cambio de trabajo dentro de la educación se relaciona en mucha mayor medida con aspectos organizativos del trabajo, mientras que la segunda variable de estudio está más vinculada a cuestiones de desarrollo profesional. Es decir, las personas que tienen intención de cambiar de puesto de trabajo (pero no de sector profesional) quieren mejorar las circunstancias específicas de su trabajo, mientras que los que optan por abandonar la educación parecen buscar nuevos intereses fuera de este ámbito laboral. Además, en el primer caso se ha observado una mayor relación con la satisfacción general con el empleo, puesto que una mayor intención de cambio se relaciona con un mayor porcentaje de insatisfacción. Esta relación se da también en el deseo de cambio de profesión, pero de manera menos rotunda (hay más porcentaje de personas que, estando satisfechas, han pensado en dejar la educación) tal y como se muestra en la tabla 5.

De forma más específica, la intención de cambio 
hacia otro puesto dentro del sistema educativo se relaciona, en gran medida, con la satisfacción con determinados aspectos como: la cantidad de horas de docencia o la cooperación con alumnado y progenitores. Por ejemplo, el $30,4 \%$ de las personas que afirman estar poco o nada satisfechas con el número de horas de docencia se han planteado un cambio de puesto, mientras que tan sólo el $8,8 \%$ de las que están bastante 0 muy satisfechas han pensado en dicho cambio. En este sentido, es relevante mencionar que se trata de una cuestión de percepción, puesto que el número medio de horas de docencia es muy similar entre los docentes satisfechos y los insatisfechos. Por otro lado, el 38,5\% de los docentes insatisfechos con la cooperación con madres y padres han pensado en cambiarse de trabajo, mientras que sólo el $11,8 \%$ de los que están satisfechos se lo han planteado. 0 tras cuestiones que inciden en el deseo de cambio son la gestión de la información en el centro o el tamaño de los grupos escolares. Nuevamente, a mayor insatisfacción, mayor deseo de cambio. El 46,7\% de las personas insatisfechas con la gestión de la información en el centro se han planteado un cambio, por el $14,5 \%$ de las que están contentas con esta cuestión en concreto.

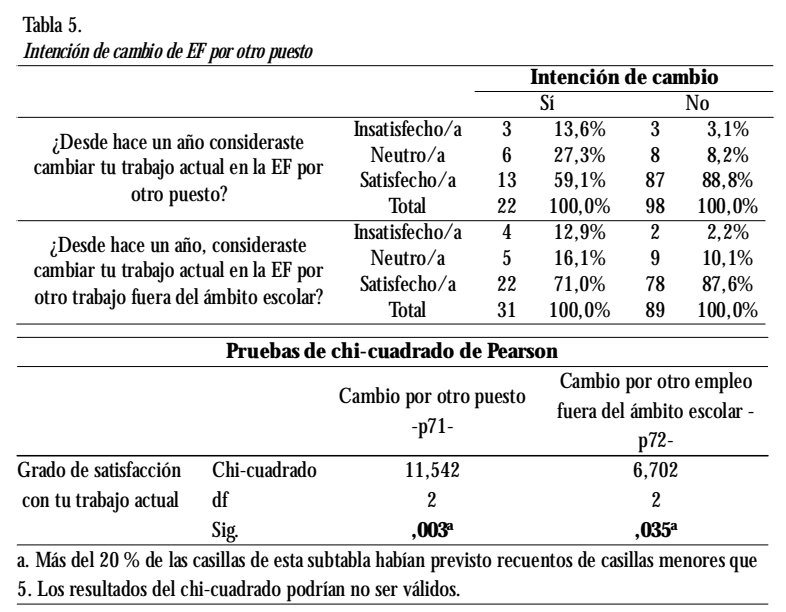

Por otro lado, los aspectos que más claramente están relacionados con la intención de cambiar de empleo y abandonar el ámbito escolar son la satisfacción con las posibilidades de influir en el trabajo y la satisfacción con las posibilidades de desarrollo profesional. En el primer caso, el 58,3\% de las personas poco 0 nada satisfechas han pensado en cambiar de empleo, cifra que decrece hasta el $20,9 \%$ en el caso de docentes bastante 0 muy satisfechos. I gualmente, el $41,7 \%$ de los docentes insatisfechos con las posibilidades de desarrollo profesional se han planteado cambiar de ámbito laboral, por el 18,3\% de los que se muestran más satisfechos. La satisfacción con el salario presenta unos valores semejantes, aunque el valor de chi-cuadrado es ligeramente inferior y hay que tratarlo con cierta cautela.

\section{Discusión y conclusiones}

Las cuestiones que se pretenden responder en este estudio se centran en el análisis del desgaste que sufren los docentes de EF en activo, concretamente en las razones que manifiestan a la hora buscar un cambio en su desempeño profesional. Así pues, se encontró que los docentes de EF estaban generalmente satisfechos con su profesión; aquellos que buscaban alternativas eran en su mayoría hombres en la mitad de su carrera profesional que estaban considerando cambios de especialidad o puestos gerenciales como una opción profesional. Los docentes de centros públicos y los que tienen otras responsabilidades además de la docencia en EF parecen más dispuestos a cambiar de puesto de trabajo.

En trabajos previos se ha encontrado un sentimiento general de satisfacción con la profesión (Eirín Nemiña, Sanmiguel-Rodríguez, y Rodríguez Rodríguez, 2020; González y Subaldo, 2015; M uñozM éndez, Gómez-M ármol y Sánchez-Alcaraz, 2017; Zurita et al., 2015), y difieren de los ofrecidos por M äkelä, Hirvensal o, Laakso y W hipp (2014) al señalar que el $39 \%$ de los docentes de EF manifiestan su deseo de abandonar la profesión. En este sentido la satisfacción con el trabajo no es tan alta y se da un importante abandono de la profesión y problemas de reclutamiento de docentes. Eirín Nemiña et al. (2020) mostraron que un $20,8 \%$ de los docentes de EF sí querían cambiar de trabajo y que lo harían por un puesto en gestión deportiva, docencia universitaria 0 deportes y coaching de ocio; e indicaron que un $1,7 \%$ no volvería a ser docente de EF y se interesan en el cambio a otra especialidad docente y la asunción de tareas de gestión. Estos resultados coinciden con los expresados en el trabajo de Gaete Silva, Castro Navarrete, Pino Conejeros y Mansilla Devia (2017). Un número muy reducido manifiesta su preferencia por la representación sindical (1), gestión deportiva y actividades de ocio-tiempo libre (1), y docencia universitaria (1). Es de destacar la diferencia entre los docentes de primaria y secundaria. Mientras los primeros suelen manifestar su deseo de pasar a ser docentes generalistas, los segundos se incli- 
nan en mayor proporción por tareas de gestión educativa. Diferencias que vienen a coincidir con las manifestadas en el trabajo de Moreira, Nascimento, Sonoo y Both (2010). Las diferencias de experiencia docente también apoyan la preferencia hacia nuevos caminos en la profesión. A medida que se cumplen años los docentes de EF manifiestan su deseo de cambio de orientación profesional (Carson, Richards, Hemphill y Templin, 2016). Por el contrario, aquellos docentes más jóvenes manifiestan en menor medida sus deseos de abandonar la EF, en la línea de lo manifestado por Perie y Baker (1997).

Al categorizar los elementos que empeoran la satisfacción docente y contribuyen a su desgaste, se aprecia una cierta discrepancia con lo recogido en la literatura. Así, suelen aparecer las condiciones extrínsecas: escasez de material, malas condiciones de las instalaciones, grupos numerosos y salario (Ávalos y Valenzuela, 2016; Buckley et al., 2004; Hancock y Scherff, 2010) como causantes de la insatisfacción de los docentes (C astro, Santiago, Villardefrancos y O tero-López, 2009; López, 2013; M entado-Labao y Castro-González, 2014). Según los resultados que se han obtenido en esta investigación, las condiciones materiales y equipamientos como la organización docente no destacan en las atribuciones de desgaste señaladas por los docentes.

Por el contrario, atribuyen de forma muy notable a su desencanto y desgaste, las dimensiones de colaboración, gestión de la escuela y especialmente la consideración hacia la EF. Si bien en la literatura también se recoge la problemática baja consideración a la labor del profesorado (A naya y López, 2014; Betoret, 2009; Hardman, 2011; M acdonald, 1995), la escasa colaboración (Hargreaves, 2019) y los problemas de gestión de la materia en el contexto escolar (Ávalos y Valenzuela, 2016). Conviene destacar la contundencia de los resultados sobre la escasa consideración y colaboración de la administración hacia la EF, y también la escasa valoración que le atribuyen las familias, siendo estos los aspectos con peor puntuación. El hecho de poder transmitir valores de respeto, esfuerzo, compañerismo, empatía, solidaridad, superación, hábitos sal udables y alternativas de ocio, conforman un conjunto de factores que inciden positivamente en la motivación y satisfacción de los docentes con su trabajo y son congruentes con lo señalado por U sán, Salavera, M erino y J arie (2018).

Se concluye que existe un cierto desgaste e insatisfacción en los docentes de EF gallegos analizados en este trabajo. Los factores que parecen determinantes y que influyen en esta situación son la escasa consideración hacia la EF por parte de las familias y la falta de apoyo e incompetencia de la administración. Cuando los docentes deciden buscar un nuevo horizonte profesional suelen optar por un cambio de especialidad y la dedicación a las tareas de gestión docente, lo que reaviva sus motivaciones docentes y profesionales.

\section{Limitaciones del estudio}

En este estudio se ha empleado un análisis descriptivo y correlacional sobre los cambios de desempeño laboral en los docentes de EF. En cuanto a las limitaciones del estudio, se deben mencionar los siguientes aspectos. El primero tiene que ver con el marcado carácter descriptivo, al tratarse de uno de los primeros estudios desarrollados en la CCAA de Galicia sobre la satisfacción laboral y el desgaste que sufre el profesorado de EF. Además, para profundizar en el grado de percepción de los docentes resultaría interesante complementar este trabajo con una perspectiva cualitativa que permitiese profundizar en una mayor comprensión del fenómeno estudiado. Al ser el cuestionario el principal instrumento de observación, nos da una «foto fija» de un determinado momento con unas circunstancias particulares. Las apreciaciones de los docentes cambian con el tiempo y en respuesta a nuevas circunstancias del trabajo, por lo que repetir el estudio y/ o complementarlo con otros instrumentos puede hacer que el diseño de la investigación sea más robusto.

También hay que señalar que este estudio ha sido elaborado con un cuestionario de autorregistro e informe. Este instrumento mide las opiniones de los participantes, en lugar de medir las manifestaciones prácticas de este constructo. La observación directa en sus contextos, centros y aulas, podría proporcionar valoraciones más objetivas de las apreciaciones de los docentes y documentar con mayor precisión los factores que influyen en su satisfacción.

\section{Referencias}

Ávalos, B. yValenzuela, J. P. (2016). Education for all and attrition/ retention of new teachers: $A$ trajectory study in Chile. International Journal of Educational Development, 49, 279-290. https:/ / doi.org/ 10.1016/ j.ijedudev.2016.03.012 
Anaya, D. y López, E. (2014). Satisfacción laboral del profesorado en 2012-13 y comparación con los resultados de 2003-04. Un estudio de ámbito nacional. Revista de Educación, 365, 96-121. http:/ / dx. doi.org/ 10.4438/ 1988-592X-RE-2014-365266

Barmby, P. (2006). Improving teacher recruitment and retention: the importance of workload and pupil behaviour. Educational Research, 48(3), 247265. 00131880600732314

Betoret, F. D. (2009). Self-Efficacy, School Resources, J ob Stressors and Burnout among Spanish Primary and Secondary School Teachers: A Structural Equation Approach. Educational Psychology, 29, 4568. 01443410802459234

http:/ / dx.doi.org/ 10.1080/

Buckley, J., Schneider, M. y Shang, Y. (2004). The effects of school facility quality on teacher retention in urban school districts. Washington, DC: $N$ ational Clearinghouse for Educational Facilities 24, 2005-132.

Carson, R. L., Richards, K. A. R., Hemphill, M. A. y Templin, T. J. (2016). Exploring the job satisfaction of late career secondary physical education teachers. Journal of Teaching in Physical Education, 35, 284-289. http:/ / dx. doi:10.1123/ jtpe. 2015-0131

Castro, C., Santiago, M. J., Villardefrancos, E. y 0 tero-López, J. M. (2009). Job dissatisfaction and burnout in secondary school teachers: student's disruptive behaviour and conflict management examined. European Journal of Education and Psychology, 2(2), 99-111.

Eirín-N emiña, R., Sanmiguel-Rodríguez, A. y Rodríguez-Rodríguez, J. (2020). Professional satisfaction of physical education teachers. Sport, Education and Society, 1-14. https:/ / doi.org/ 10.1080/ 13573322.2020.1816540

Gaete Silva, A., Castro Navarrete, M., Pino Conejeros, F. y Mansilla Devia, D. (2017). Abandono de la profesión docente en Chile: Motivos para irse del aula y condiciones para volver. Estudios pedagógicos (Valdivia), 43 (1), 123-138. https:/ / doi.org/ 10.4067/ S0718-07052017000100008

Gaudreault, K. L. y Woods, A. M. (2013). Factors leading to career frustration and exit: A case of a veteran physical educator. Journal of Physical Education and Sports Management, 4(4), 51-61. https:/ / doi. org/ 10.5897/ J PESM 2012.0159
González, J. y Subaldo, L. (2015). O piniones sobre el desempeño docente y sus repercusiones en la satisfacción profesional y personal de los profesores. Educación, 24(47), 90-114.

Hancock, C. B. y Scherff, L. (2010). W ho will stay and who will leave? Predicting secondary English teacher attrition risk. Journal of Teacher Education, 61(4), 328-338. https:/ / doi.org/ 10.1177/ 0022487110372214

Hardman, K. (2011). G lobal issues in the situation of physical education in schools. Contemporary issues in physical education, 11-29.

Hargreaves, A. (2019). Teacher collaboration: 30 years of research on its nature, forms, limitations and effects. Teachers and Teaching, 25(5), 603-621. ht t ps: / / doi .org/ $10.1080 /$ 13540602.2019.1639499

Ingersoll, R. y Merrill, L. (2010). W ho's teaching our children? Educational Leadership, 67(8), 14-20. Judge, T. A., Thoresen, C. J., Bono, J. E. y Patton, G. K. (2001). The job satisfaction-job performance relationship: A qual itative and quantitative review. Psychological Bulletin, 127(3), 376-407. https:// doi.org/ 10.1037/ 0033-2909.127.3.376

Karsenti, T. y Collin, S. (2013). W hy are new teachers leaving the profession? results of a Canada-wide survey. Education, 3(3), 141-149.

Keigher, A. (2010). Teacher Attrition and Mobility: Results From the 2008-09 Teacher Follow-up Survey (NCES 2010-353). U.S. Department of Education. Washington, DC: National Center for Education Statistics. Retrieved [10/ 09/2018] from http:/ / nces. ed. gov/ pubsearch.

Locke, E. A. (1976). The nature and causes of job satisfaction. $\mathrm{H}$ andbook of industrial and organizational psychology.

López, A. (2013) Satisfacción profesional, marco de condiciones para la enseñanza y calidad de las clases de educación física. Un estudio de caso en profesores de una municipalidad. Acción motriz, 10, 59-75.

MacBeath, J. (2012). Future of teaching profession. Cambridge: Educational International Research Institute and University of Cambridge.

M äkelä, K., Hirvensal o, M., Laakso, L. y W hipp, P. R. (2014) Physical education teachers in motion: an account of attrition and area transfer. Physical Education and Sport Pedagogy, 19 (4), 418-435. https:/ / doi.org/ 10.1080/ 17408989.2013.780590 Martínez, L. y González, G. (2016). Docentes de 
carne y hueso: Enseñar con el cuerpo. Ágora para la Educación Física y el deporte, 18(3), 259-275

Macdonald, D. (1995). The role of proletarization in physical education teacher attrition. Research Quarterly for Exercise and Sport, 66(2), 129-141. ht t p s: / / do i.org/ 10.1080 / 02701367.1995.10762220

Mentado-Labao, T. y Castro-González, L. (Coord.) (2014). Satisfacción profesional del profesorado de Educación Primaria. VI Seminario internacional del Observatorio Internacional de la Profesión D ocente. Barcelona. Universidad de Barcelona.

Mihans, R. (2008). Can teachers lead teachers. Phi Delta Kappan, 89 (10), 762- 765. https:/ / doi. org/ $10.1177 / 003172170808901016$

Mohr, D. J. yTownsend, S. J. (2001). In the beginning: $\mathrm{N}$ ew physical education teachers' quest for success. Teaching Elementary Physical Education, 12(4), 9-11.

Moore, C. M. (2012). The role of school environment in teacher dissatisfaction among U.S. public school teachers. SAGE open, 2(1), 1-16. https:/ / doi. org/ $10.1177 / 2158244012438888$

Moreira, H. D. R., Nascimento, J. V. D., Sonoo, C. N. y Both, J. (2010). Q ualidade de vida do trabalhador docente em Educação Física do estado do Paraná, Brasil. Revista Brasileira de Cineantropometria \& D esempenho H umano, 12 (6), 435442. https:// doi.org/ 10.5007/ 19800037.2010v12n6p435

Moreira, H. , Sparkes, A. C.Y Fox, K. (1995). Physical education teachers and job commitment. $A$ preliminary analysis. European Physical Education Review, 1(2), 122-136. https:/ / doi.org/ 10.1177/ $1356336 \times 9500100204$

M uñoz-M éndez, A., Gómez-M ármol, A. y SánchezAlcaraz, B.J. (2017) Satisfacción laboral en los docentes de educación infantil, primaria y secundaria. Gestión de la educación, 7(1), 161-177.

Perie, M. y Baker, D. P. (1997). Job satisfatcion among America's teachers: Effects of workplace conditions, background characteristics, and teacher compensation. Washington, DC: U.S. Department of Education.

Perrachione, B. A., Rosser, V. J. y Petersen, G. J. (2008). W hy do they stay? Elementary teachers' perceptions of job satisfaction and retention. Professional Educator, 32(2), 25-41.

Pinder, C. C. (1998). Work motivation in organizational behavior. Upper Saddle River, NJ: Prentice Hall. Richards, K, A. R. y Templin, T. J. (2012). Toward a multidimensional perspective on teacher-coach role conflict. Quest, 64(3), 164-176. https:// doi.org/ 10.1080/ 00336297.2012.693751

Sargent, T. y Hannum, M. (2005). Keeping tachers happy: Job satisfaction among primary school teachers in rural Northwest China. Comparative Education Review, 49(2), 173-204. https:/ / doi.org/ $10.1086 / 428100$

Skaalvik, E. M. y Skaalvik, S. (2011). Teacher job satisfaction and motivation to leave the teaching profession: Relations with school context, feeling of belonging, and emotional exhaustion. Teaching and Teacher Education, 27, 1029-1038. https:/ / doi.org/ 10.1016/ j.tate.2011.04.001

Smith, D. y Leng, G. W. (2003). Prevalence and sources of burnout in Singapore secondary school physical education teachers. Journal of Teaching in Physical Education, 22(2), 203-218. https:/ / doi.org/ 10.1123/ jtpe.22.2.203

Spector, P. E. (1996). Industrial and organizational psychology. N ew York: W iley.

U sán, P., Salavera, C., M erino, A. y Jarie, L. (2018). Satisfacción de necesidades psicológicas y orientaciones de meta en profesorado de Educación Física hacia sus al umnos. Retos. N uevas tendencias en Educación Física, Deporte y Recreación, 33, 50-53.

Weiss, H. M. (2002). Deconstructing job satisfaction: Seperating evaluations, beliefs and affective experiences. Human Resource Management Review, 12, 173-194. http:/ / doi:10.1016/ S10534822(02)00045-1

Zembylas, M. y Papanastasiou, E. (2006). Sources of teacher job satisfaction and dissatisfaction in Cyprus. Compare, 36(2), 229-247. https:/ / doi. org/ 10.1080/03057920600741289

Zurita, F., Rojas, M., Linares, D., López, C. J. Martínez, A. y Castro, M. (2015). Satisfacción laboral en el profesor de educación física de Cienfuegos (Cuba). Revista deCiencias Sociales (RCS), 21(2), 261-274.

\section{(Footnotes)}

${ }^{1}$ Estos porcentajes se refieren a las personas que han respondido «Ś́ » a la pregunta planteada (en este caso, si han pensado en cambiar de puesto de trabajo dentro de la educación).

2 Porcentajes referidos a las per sonas que han dicho que en el último año consideraron alguna vez cambiar de empleo por otro fuera de la educación. 